\title{
Embryonal tumor with abundant neuropil and true rosettes: Morphological, immunohistochemical, ultrastructural and molecular study of a case showing features of medulloepithelioma and areas of mesenchymal and epithelial differentiation
}

\author{
Anna Maria Buccoliero, ${ }^{1}$ Francesca Castiglione, ${ }^{2}$ Duccio Rossi Degl'Innocenti, ${ }^{2}$ \\ Alessandro Franchi, ${ }^{2}$ Milena Paglierani, ${ }^{2}$ Massimiliano Sanzo, ${ }^{3}$ Valentina Cetica, ${ }^{4}$ Laura Giunti, ${ }^{5}$ \\ Iacopo Sardi, ${ }^{4}$ Lorenzo Genitori ${ }^{3}$ and Gian Luigi Taddei ${ }^{2}$
}

${ }^{1}$ Department of Biomedicine, Careggi Hospital, ${ }^{2}$ Department of Human Pathology and Oncology, University of Florence, and Divisions of ${ }^{3}$ Neurosurgery, ${ }^{4}$ Oncoematology and ${ }^{5}$ Medical Genetic, Anna Meyer Children's Hospital, Florence, Italy

Embryonal tumors are a group of malignant neoplasms that most commonly affect the pediatric population. Embryonal tumor with abundant neuropil and true rosettes is a recently recognized rare tumor. It is composed of neurocytes and undifferentiated neuroepithelial cells arranged in clusters, cords and several types of rosettes in a prominent neuropil-rich background. We describe a new case of this tumor. The patient, a 24-month-old female infant, was referred to the Meyer Children's Hospital with a history of right brachio-crural deficit associated with occasional episodes of headache and vomiting. Computed tomography scan and MRI revealed a large bihemispheric mass. The patient underwent two consecutive surgeries. The resultant surgical resection of the tumor was macroscopically complete. The postoperative period was uneventful. On light microscopy the tumor showed a composite morphology: embryonal tumor with abundant neuropil and true rosettes (specimen from the first surgery); medulloepithelioma with mesenchymal and epithelial areas (specimen from the second surgery). The immunohistochemistry evidenced the heterogeneous (neuronal,

Correspondence: Anna Maria Buccoliero, MD, PhD, Department of Biomedicine, Careggi Hospital, Florence, V.le GB. Morgagni, 85, 50134 Florence, Italy. Email: ambuccoliero@unifi.it

Received 31 March 2009; revised and accepted 13 May 2009. mesenchymal and epithelial) immunoprofile of tumoral cells. By real-time polymerase chain reaction (RT-PCR), the PTEN gene expression in the tumor was lower than in the five non-neoplastic brain tissues used as control. Mutation analysis did not show any variation in INI-1 and PTEN sequence while P53 analysis showed the presence of homozygote P72R variation. Fluorescent in situ hybridization analysis showed polysomy of chromosome 2 while amplification of $N-M Y C$ was not detected. Owing to the rarity of embryonal tumor with abundant neuropil and true rosettes, each new case should be recorded to produce a better clinical, pathological and molecular characterization of this lesion.

Key words: brain, central nervous system, embryonal tumor, p53, pnet.

\section{INTRODUCTION}

Embryonal tumors are a group of malignant neoplasms that most commonly affect the pediatric population. The latest edition of the World Health Organization (WHO) classification of CNS tumors distinguishes three main histological entities: medulloblastoma and its variants (desmoplastic medulloblastoma, medulloblastoma with extensive nodularity, anaplastic medulloblastoma and large cell medulloblastoma), CNS primitive neuroectodermal 
tumor (PNET) ant its variants (CNS neuroblastoma, CNS ganglioneuroblastoma, medulloepithelioma and ependymoblastoma) and atypical teratoid/rhabdoid tumor (AT/ RT). ${ }^{1}$

In 2000, Eberhart et al. described a rare pediatric embryonal tumor called pediatric neuroblastic tumor containing abundant neuropil and true rosettes. ${ }^{2}$ This tumor combines the features of neuroblastoma and ependymoblastoma. It is morphologically characterized by undifferentiated neuroepithelial cells arranged in clusters, cords and several types of rosettes (i.e. ependymoblastic, Homer-Wright, Flexner-Wintersteiner and perivascular) in a neuropil-rich background. Although a number of such cases have been described, this entity has not been considered a distinct neoplasm (it has been believed to be just "an unusual PNET") in the WHO classification of CNS tumors and also its appropriate nomenclature remains to be decided (pediatric/embryonal/neuroblastic tumor with abundant neuropil and true rosettes). ${ }^{1}$

Actually, it is sometimes complex to categorize the tumors, particularly in case of neoplasms showing composite morphology.

Herein, we describe a case of embryonal tumor with abundant neuropil and true rosettes showing features of medulloepithelioma and areas of mesenchymal and epithelial differentiation. The morphological, immunohistochemical, ultrastructural and molecular (P53, PTEN, $I N I-1, N-M Y C)$ traits of this tumor are described.

\section{CASE REPORT}

A 24-month-old female infant was referred to Meyer Children's Hospital with a history of right brachio-crural deficit associated with occasional episodes of headache and vomiting. A CT scan revealed a large bihemyspheric mass with calcifications. MRI was performed as well and this documented a lesion enhanced by gadolinium with solid and cystic components (Fig. 1). Due to the dimensions of the tumor and its rich vascularisation, the patient underwent two consecutive surgeries spaced by 1 week (left parietooccipital and right parieto-occipital surgical approaches). The resultant surgical resection of the tumor was macroscopically complete. The postoperative period was uneventful with a good motor function recovery. Spinal imaging and CSF cytology were negative. Thus, the patient was treated according to the institutional protocol for infant CNS PNET which consists of high doses of chemotherapy and autologous stem cell rescue followed by tailored radiotherapy. Her treatment course passed without complication and she was discharged without relevant neurologic signs. As of the patient's last follow-up at 10 months, she was doing well with a Karnofsky performance status score of $80 \%$.

\section{METHODS}

From the fresh surgical specimens of each surgery we selected a fragment macroscopically representative of the tumor. Successively, we cut it in half: from one half several 5- $\mu \mathrm{m}$ frozen sections stained with HE were obtained to verify the adequacy of the specimen selected for molecular studies (presence of pathological tissue only); the other half was immersed in RNAlater ${ }^{\mathrm{TM}}$ (QIAGEN, Valencia, CA, US) and kept overnight at $+4^{\circ} \mathrm{C}$ and finally stored at $-80^{\circ} \mathrm{C}$ until molecular analysis (RT-PCR and gene sequencing). Total RNA was isolated with 6100 Nucleic Acid PrepStation and $500 \mathrm{ng}$ of RNA were subject to reverse transcription to cDNA using High Capacity cDNA Archive Kit (Applied Biosystems, Foster City, CA, US). Real-time quantitative PCR amplification for PTEN gene was routinely executed using the ABI PRISM 7000 Sequence Detector System (Applied Biosystems). PCR products for PTEN were detected using gene-specific primers and probes labeled with reporter day FAM (Assay on Demand, Applied Biosystems). A series of five nonneoplastic brain tissues were used as control samples. $g A P D H$ was used as endogenous control gene for normalization. For gene sequencing, genomic DNA from tumor tissues of specimens from both surgeries, were extracted with QIAamp DNA Kit (QIAGEN, Milan, Italy) according to the manufacture's protocol. DNA concentrations were determined using spectrophotometer GeneQuant II (Pharmacia, Cambridge, UK). The complete coding sequence and flanking exon-intron borders of the INI-1 (exons 1-9), PTEN (exons 1-9) and P53 (exons 2-11) genes were investigated by direct sequencing. PCR primers are available upon request. PCR products were sequenced by $\mathrm{ABI}$ Prism $^{\circledR} 3130$ Genetic Analyzer (Applied Biosystems).

Several additional fresh fragments from the first surgery macroscopically representative of the tumor were fixed in $2.5 \%$ glutaraldehyde and processed for electron microscopy by conventional method (ultrathin sections were stained using uranyl acetate and lead citrate, then observed with a Philips 410 LS transmission electron microscope).

The remaining tumor specimens from both surgeries were routinely formalin-fixed and paraffin-embedded. Histological sections were stained with HE for histomorphological evaluation. Further $5-\mu \mathrm{m}$ sections of the most representative sample were mounted on electrostatic slides and used for the standard immunohistochemical and dual color fluorescence in situ hybridization (FISH) analysis. Immunohistochemical studies were performed using the standard streptavidin-biotin technique and commercially available reagents. The primary antibodies used were against: smooth muscle actin (clone 1A4, Cell Marque Corporation, Rocklin, CA, US), muscle-specific actin (clone HHF35, Cell Marque), synaptophysin (SP, polyclonal, Cell 


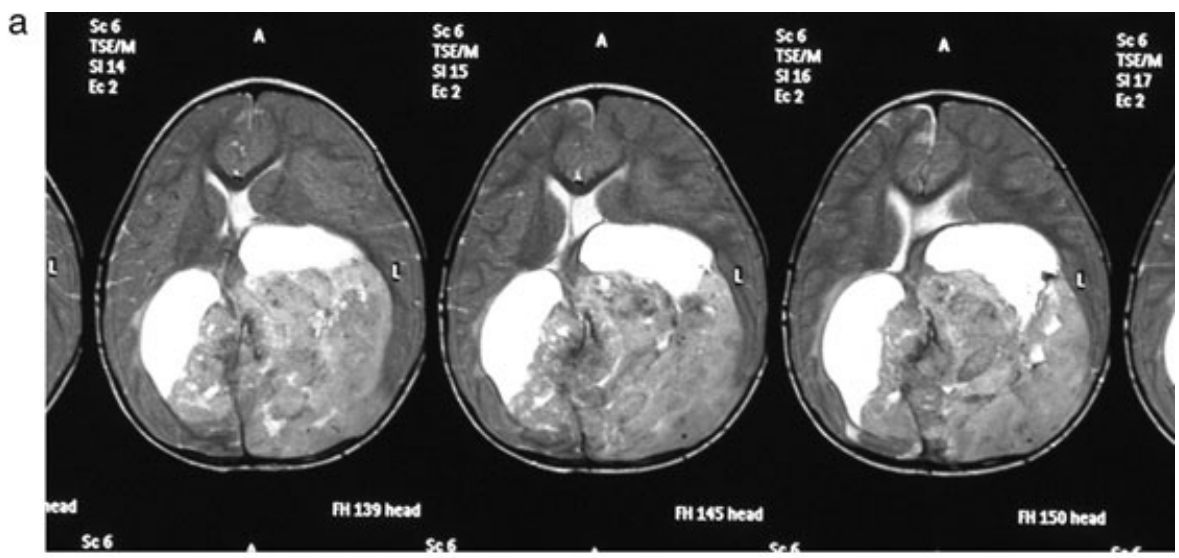

Fig. 1 (a) MRI axial T2-weighted image shows a large tumor involving the left parietal and occipital lobe and extending over the midline to the upper part of the controlateral cerebral hemisphere. The MRI signal is iso-hyperintense with small areas of hyperintensity. (b) MRI axial T1-weighted gadolinium-enhanced image shows the disomogeneous contrast medium-enhancing and iso-hyperintense signal of the tumor. (c) MRI coronal T1-weighted gadolinium-enhanced image shows the midline shift on the lower part of parieto-occipital lobe without invasion of the lower part of the controlateral cerebral hemisphere, with disomogeneous contrast-enhancement as well as MR signal that appear iso-hyperintense.
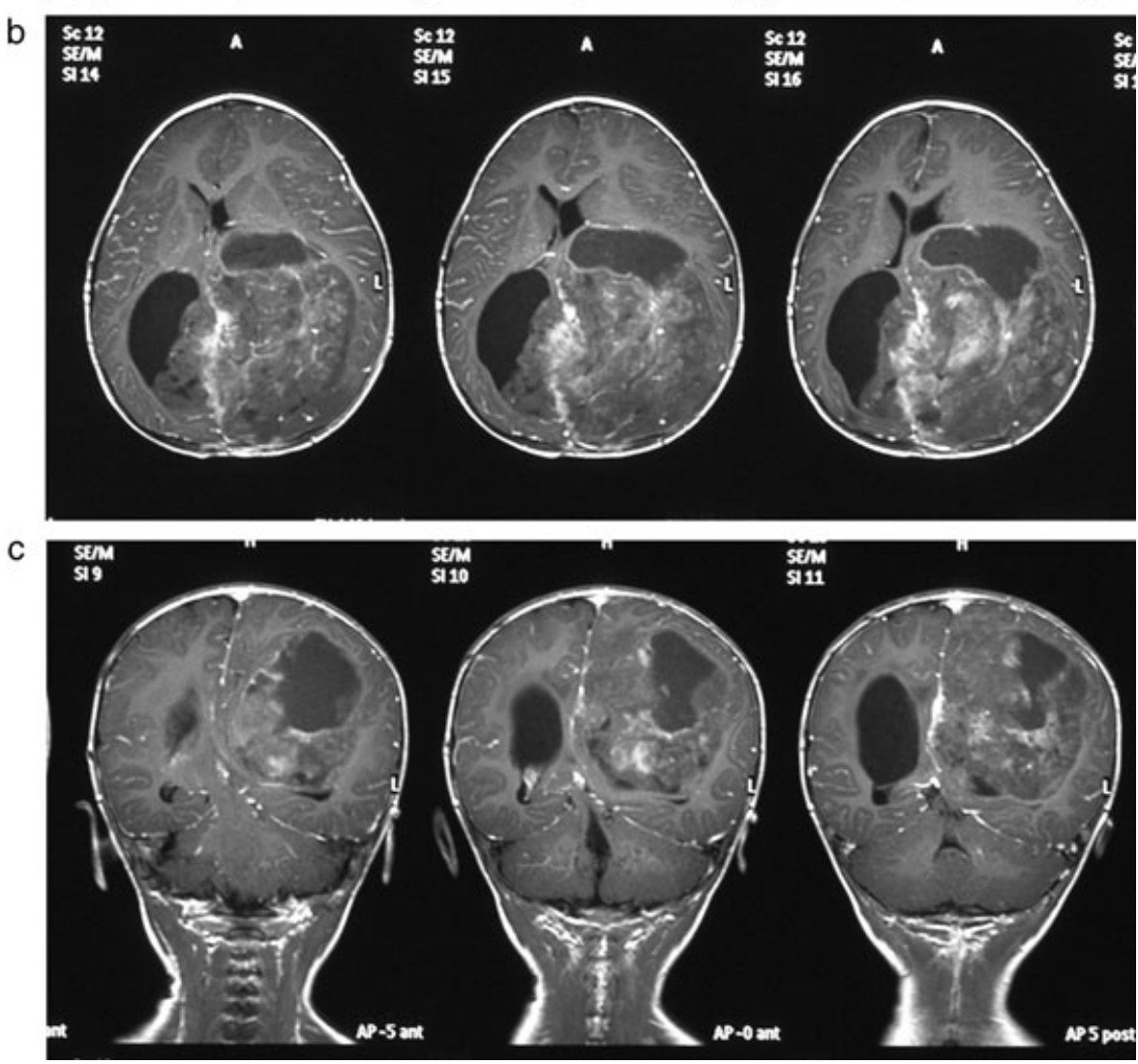

Marque), neurofilaments (NF, clone 2F11, Cell Marque), desmin (clone DE-R-11, Ventana Medical Systems, Tucson, AZ, US), cytokeratins (clone AE1, Ventana; clone AE3, Ventana; clone CAM 5.2, BD Biosciences, Becton Dickinson, San Jose, CA, US), p53 (clone DO-7, Ventana), BAF47 (INI-1, clone 25/BAF47, BD Biosciences) and Ki-67 (clone Mib-1, Dako A/S, Glostrup, Denmark).

For detection of the $N-M Y C$ oncogene, located at 2p24 region, we used the $\mathrm{LSI}^{\circledR} N-M Y C$ (2p24)/ CEP® 2 (2p11.1q11.1) Dual Color Probe Set (Abbott Molecular Inc., Abbott Park, IL, USA). The LSI N-MYC (2p24) probe was used to detect the $N-M Y C$ oncogene copy number to assess the amplification status of the $N-M Y C$ gene. The
CEP 2 probe serves as a control for the ploidy level for chromosome 2. For each specimen, signals were enumerated in 100 non-overlapping nuclei. Specimens were considered amplified when they demonstrated a $N-M Y C / C E P$ 2 ratio greater than 2 .

\section{RESULTS}

On light microscopical examination, the tumor specimens from the two surgeries were very different. Microscopic sections from the first surgery revealed wide neuropil-rich areas containing neurocytic elements and hypercellular regions composed by small cells with round-to-oval nuclei,

(C) 2009 Japanese Society of Neuropathology 
scanty cytoplasm and indistinct cell borders (Fig. 2). The lesion showed numerous rosettes (perivascular, ependymoblastic and Homer-Wright) (Fig. 3). Mitotic figures and prominent apoptotic bodies were frequently observed in highly cellular areas and in the rosettes. Necrosis and hem-
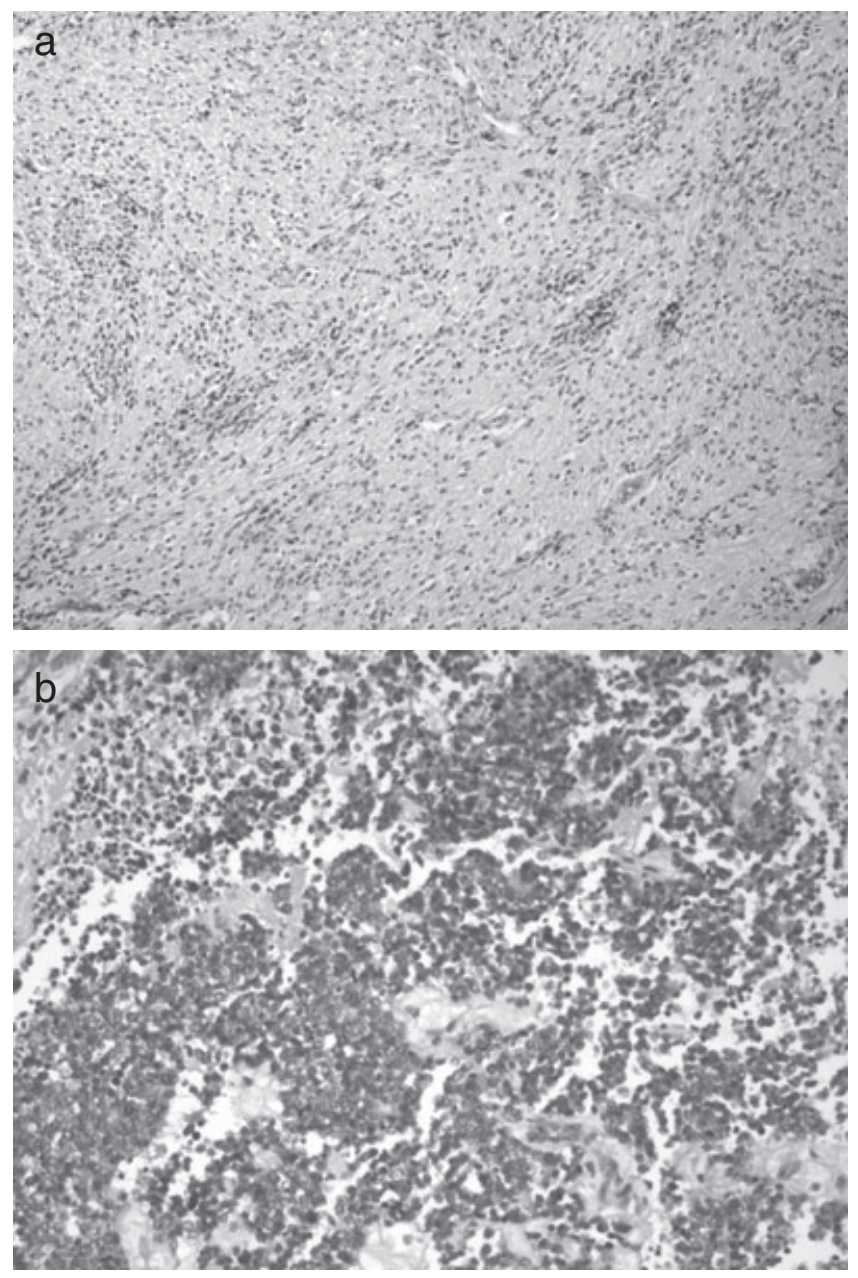

Fig. 2 Microscopic sections from the first surgery revealed neuropil-rich areas containing neurocytic elements (a) and hypercellular regions composed by small undifferentiated cells (b). HE; original magnification: (a) $\times 100$, (b) $\times 200$. orrhage were present. The tumor border was sharply demarcated from the non-neoplastic nervous parenchyma. By immunohistochemistry, there was a diffuse positivity to SP (Fig. 4). Neuropil-rich areas were positive to NF. The undifferentiated cells (hypercellular regions and rosettes) overexpressed 553 protein. The Mib-1 proliferation index ranged from $3 \%$ in the neuropil-rich areas to $80 \%$ in the areas of high cellularity and in the rosettes. The tumor cells exhibited diffuse INI-1 nuclear staining. The sample for ultrastructural analysis included areas from the neuropilrich regions, and showed neoplastic cells with round, often indented nuclei with clumped chromatin, and cytoplasm containing few organelles and abundant glycogen. Interspersed between neoplastic cells there were numerous processes of varying diameter, which contained intermediate filaments, microtubules, and few putative neurosecretory granules (Fig. 5).

Microscopic sections from the second surgery revealed a composite tumor without neuropil-rich areas. There were hypercellular undifferentiated zones composed by small cells mainly organized in a perivascular fashion, neural

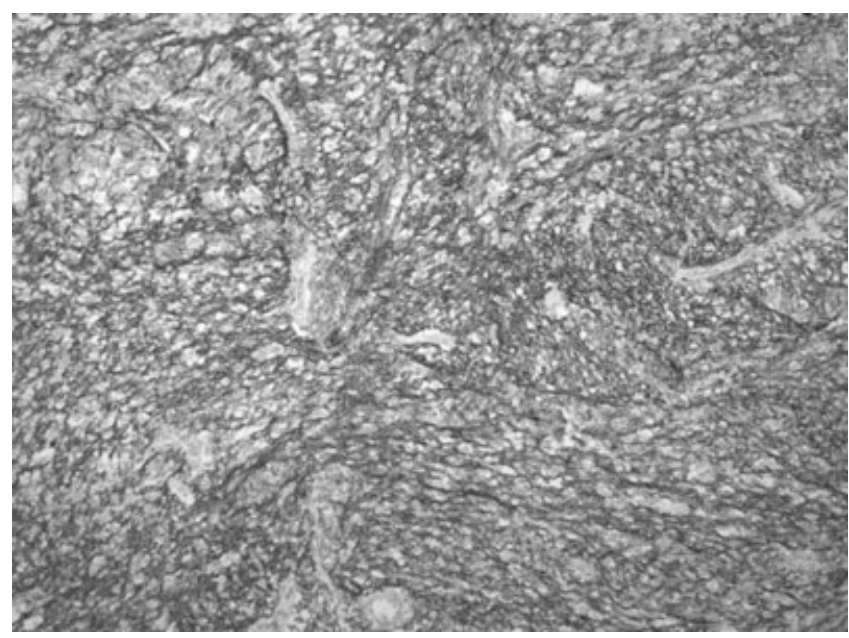

Fig. 4 Immunoistochemistry (specimen from first surgery): diffuse synaptophysin positivity. original magnification: $\times 100$.
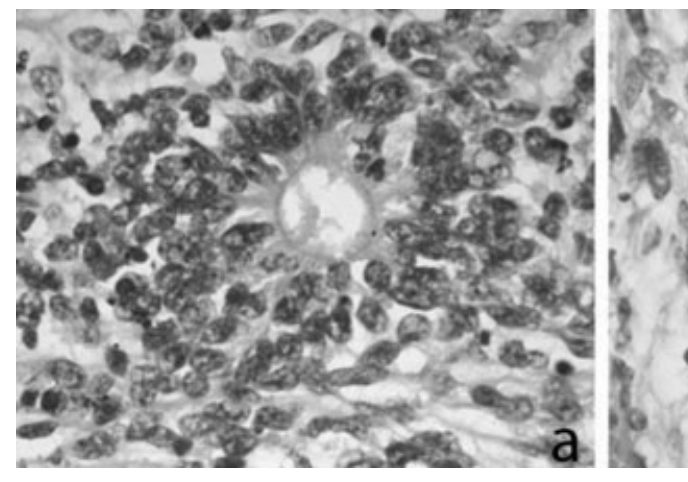

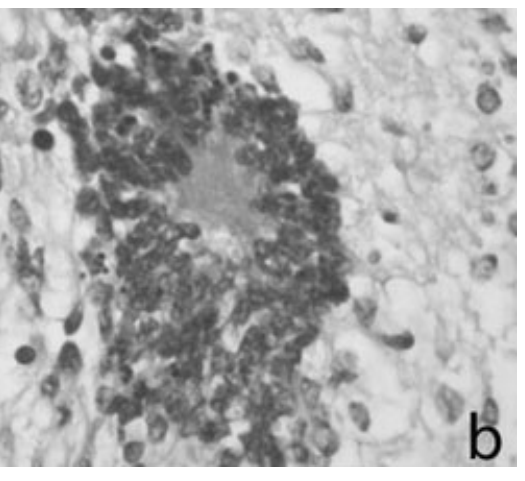

Fig. 3 The lesion (specimen from first surgery) showed numerous rosettes, i.e. ependymoblastic (a) and Homer-Wright (b). HE; original magnification: $(\mathrm{a}, \mathrm{b}) \times 200$.

(C) 2009 Japanese Society of Neuropathology 


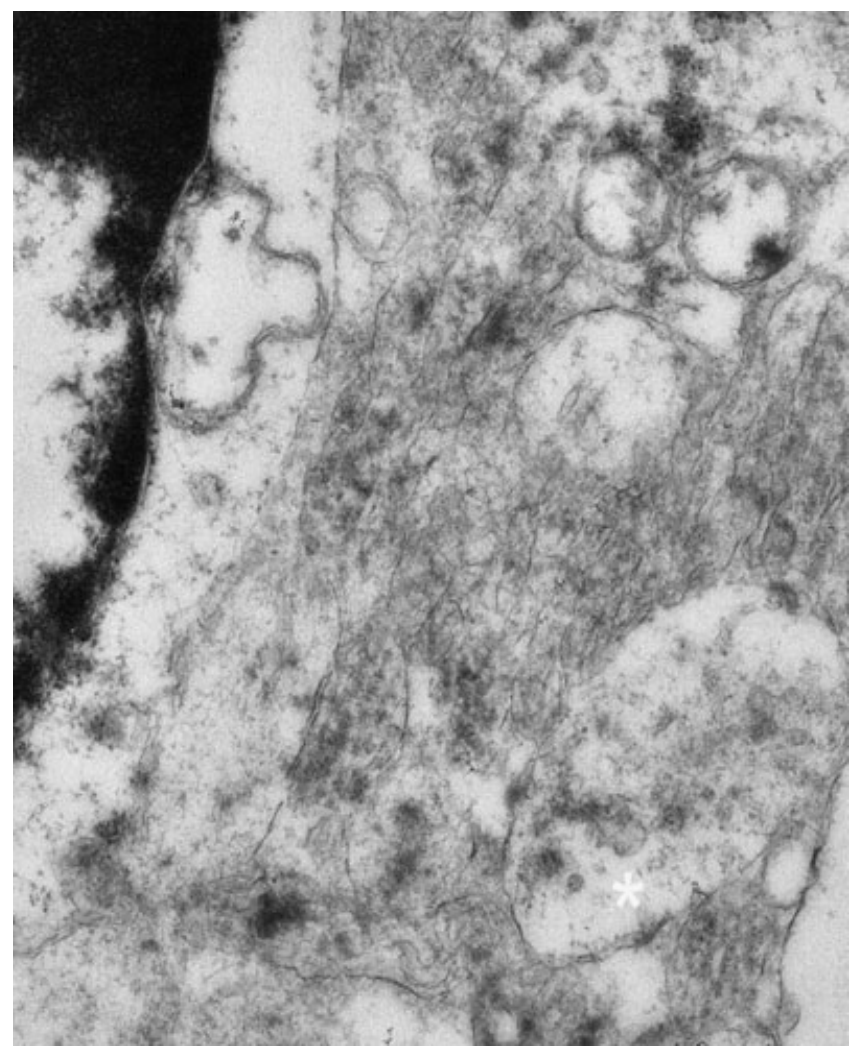

Fig. 5 Processes of varying diameter, containing intermediate filaments and few putative neurosecretory vescicles (asterisk). Original magnification $\times 14000$. tube-like structures delimited by pseudo-stratified cylindrical cells with a pseudo-papillary and trabecular arrangement and sarcomatoid areas composed by elongated cells containing medium-sized nests of epithelioid cells (Fig. 6). Analogously to the specimens from the first surgery, there were necrotic-hemorrhagic areas and the tumor border was sharply demarcated. By immunohistochemistry, the undifferentiated areas were NF-positive, the neural tubelike structures were NF- and p53-positive, the sarcomatoid areas were focally actin-positive and the epithelioid nests were AE1- and CAM 5.2-positive (Fig. 7). The Mib-1 proliferation index ranged from $10 \%$ in the sarcomatoid and epithelioid areas to $80 \%$ in areas of high cellularity and in the neural-tube like structures. The tumor cells exhibited diffuse INI-1 nuclear staining.

Morphologic and ultrastructural features along with immunohistochemical results were consistent with the diagnosis of embryonal tumor with abundant neuropil and true rosettes showing features of medulloepithelioma and areas of mesenchymal and epithelial differentiation.

By RT-PCR, the gene expression of PTEN in the first and in the second surgical specimens was lower than the $P T E N$ expression in non-neoplastic brain tissues (respectively 0.12 times and 1.73 times) using $g A P D H$ as control gene for normalization.

Mutation analysis did not show any variation in INI-1 and PTEN sequences while P53 analysis showed the presence of homozygote $\mathrm{P} 72 \mathrm{R}$ variation in both specimens (Fig. 8).
Fig. 6 Microscopic sections from the second surgery revealed a composite tumor: neural tube-like structures delimited by pseudo-stratified cylindrical cells $(\mathrm{a}, \mathrm{b})$, sarcomatoid areas composed by elongated cells containing medium-sized nests of epithelioid cells $(\mathrm{a}, \mathrm{c})$ and hypercellular undifferentiated zones composed by small cells mainly organized in a perivascular fashion (d). HE; original nagnification: $(\mathrm{a}, \mathrm{b}, \mathrm{d}) \times 100$; (c) $\times 200$.
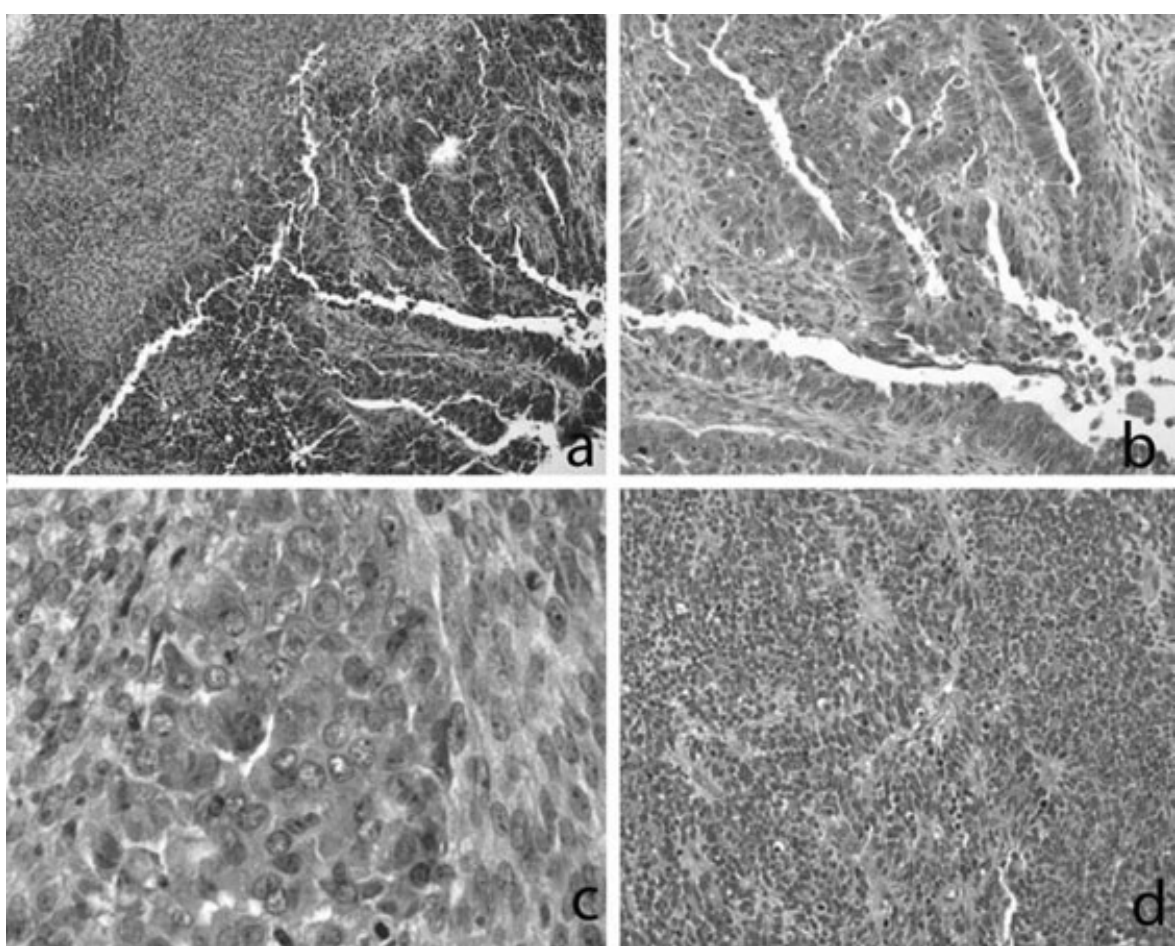

(C) 2009 Japanese Society of Neuropathology 

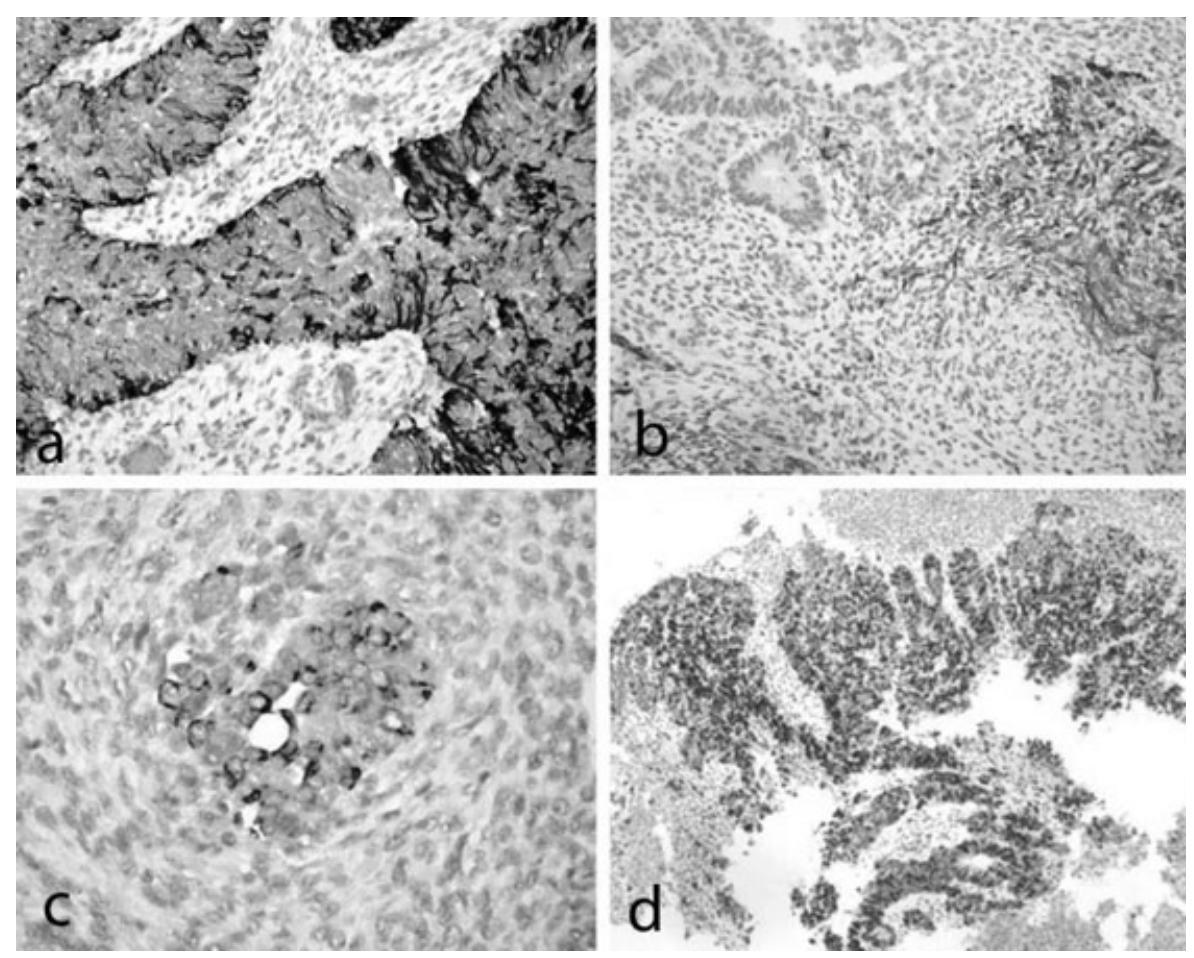

Fig. 7 Immunoistochemistry (specimen from second surgery): NF- (a) and p53-(d) positive neural tube-like structures; focal positivity to HHF35 in sarcomatoid areas (b); Cam 5.2-positive epithelioid nests (c). Original magnification: $(\mathrm{a}, \mathrm{b}) \times 100$; (c) $\times 200$; (d) $\times 50$.

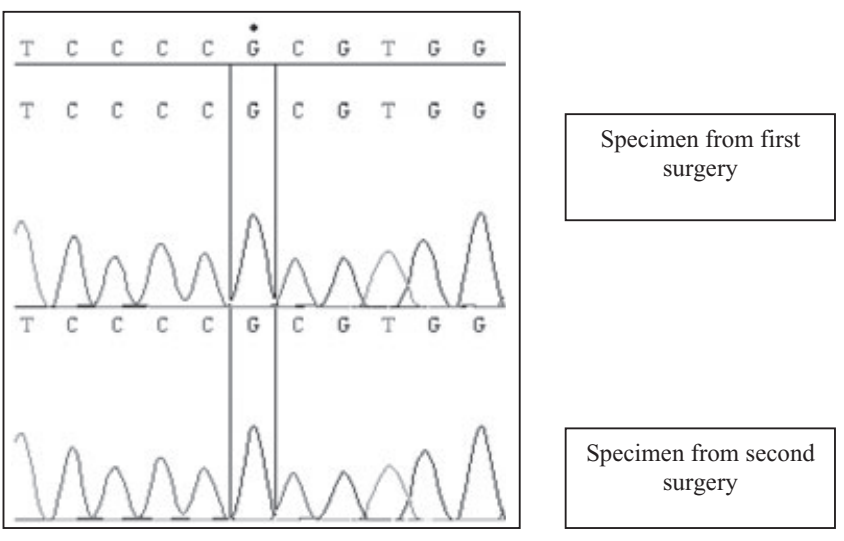

Fig. 8 P53 mutation analysis showed the presence of homozygote $\mathrm{P} 72 \mathrm{R}$ variation in both lesions.

Fluorescent in situ hybridization analysis showed polysomy of chromosome 2 in both samples, while amplification of $N-M Y C$ was not detected (Fig. 9).

\section{DISCUSSION}

Embryonal brain tumors are unusual neoplasms which are more frequent in the pediatric age-group. On the basis of their distinctive morphological, immunohistochemical, histogenetic, and molecular characteristics, they are classified as medulloblastoma, CNS PNET and AT/RT. All embryonal tumors are categorized as WHO grade IV due to the aggressive biological behavior. ${ }^{1}$ Medulloblastomas are the

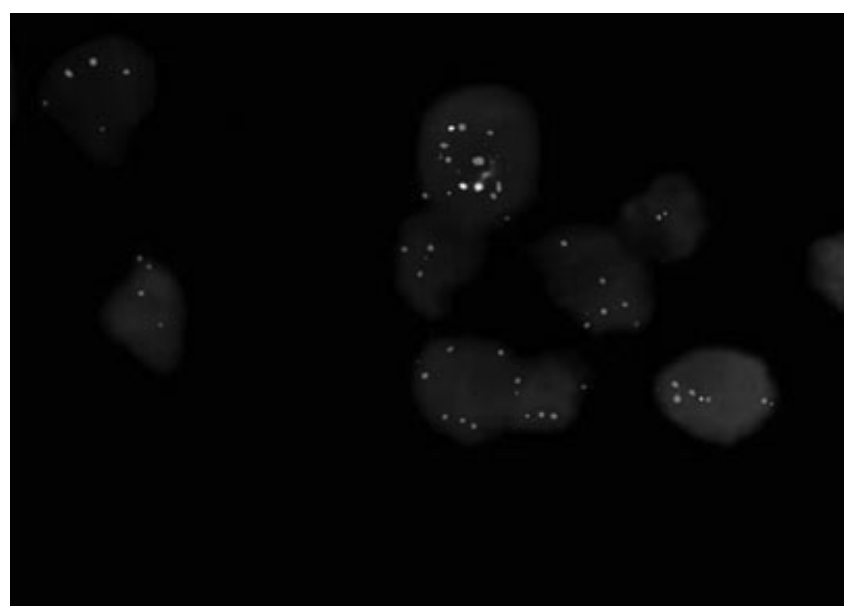

Fig. 9 Dual-color fluorescent in situ hybridization analysis (sample from first surgery) showing polysomy of chromosome 2 (orange signals); amplification of $N-M Y C$ (green signals) was not detected.

most common and constitute $\approx 20 \%$ of all primary pediatric brain tumors. ${ }^{3}$ Embryonal tumor with abundant neuropil and true rosettes is a recently recognized embryonal tumor which in the past was frequently diagnosed as ependymoblastoma due to the presence of endymoblastic rosettes. ${ }^{4}$ Recently a number of cases of this tumor have been referred, confirming the conviction that it is a distinct embryonal tumor. ${ }^{5}$ All reported cases have been in very young children with a female predominance. In the majority of the cases the lesions were large and supratentorial. 
The outcome was commonly poor despite intensive treatments. ${ }^{4,6}$ Our case is typical with regard to age, sex, size and localization, whereas it is extraordinary with regard to its singular composite morphology: embryonal tumor with abundant neuropil and true rosettes and medulloepithelioma. Indeed, contrary to the areas of epithelial and mesenchymal differentiation which are sometimes described in this tumor, ${ }^{5}$ the observation of medulloepitheliomatous areas has never been reported. Actually, it is well known as embryonal tumor, such as other neuroepithelial tumors may be polyphenotypic as a result of metaplastic-like processes or as consequence of divergent differentiation of tumoral stem cells. ${ }^{7,8}$

Due to the frequent presence in the embryonal tumor with abundant neuropil and true rosettes of ependymomastic rosettes, ependymoblastoma is the main differential diagnosis of this tumor and vice versa. In regard to this Judkins and Ellison reviewed a group of 14 ependymoblastomas diagnosed in their institution and stated that the initial diagnosis of ependymoblastoma was never confirmed. In particular eight $(57 \%)$ of these 14 ependymoblastomas were reclassified as embryonal tumor with abundant neuropil and true rosettes. ${ }^{4}$

From a molecular point of view, we did not document in our case $N-M Y C$ amplification (detected in more aggressive neuroblastomas and in large cell/anaplastic medulloblastomas) and mutations of the INI-1 (typical of AT/RT) gene., ${ }^{3,-11}$ This last conclusion was also confirmed by the diffuse INI-1 nuclear immunostaining. The FISH analysis showed case extra copies of chromosome 2 in our case. These results are in agreement to that reported by Fuller $e t$ $a l .^{5,12,13}$ Interestingly, it has been suggested that the chromosome 2 polysomy could differentiate this tumor from other embryonal tumors. ${ }^{5,12,13}$ Through gene sequencing, we identified a variation in the P53 gene in both surgical specimens. P72 variant is located in a proline-rich domain of P53 gene ${ }^{14}$ and it produces marked changes in the primary structure and biological activity of p53. Epidemiological studies have shown that differences in the polymorphism may affect the susceptibility of an individual to different tumors, even if at present there are still several controversial results in the literature..$^{15-17}$ Interestingly, immunohistochemical analysis evidenced a positive nuclear reaction at the level of the undifferentiated cells and in medulloepithelioma areas, whereas the neurocyticlike cells localized in the neuropil-rich zones as well the mesenchymal and epithelial areas, were completely negative. Based on the observation that the immunodetection of p53 correspond to the P53 gene mutation, we could suppose a biclonal molecular profile in our lesion (undifferentiated and medulloepitheliomatous areas $v s$. neuropil-rich, mesenchymal and epithelial areas). PTEN gene is one of the most commonly lost tumor suppressors in human cancer. In CNS tumors, genetic inactivation of PTEN frequently occurs in glioblastoma. ${ }^{18}$ In our case, despite PTEN expression as evaluated by RT-PCR, resulted slightly lower than non neoplastic tissue, gene sequencing did not provide evidence of PTEN alterations.

Owing to the rarity of embryonal tumor with abundant neuropil and true rosettes, each new case should be recorded to produce a better clinical, pathological and molecular characterization of this lesion.

\section{REFERENCES}

1. Lopes MBS, Wiestler OD, Stemmer-Rachamimov AO, Sharma MC. Tuberous complex ad subependymal giant cell astrocytoma. In: Louis DN, Ohgaki H, Wiestler OD, Cavenee WK, eds. WHO Classification of Tumours of the Central Nervous System, 4th edn. Lyon: IARC Press, 2007; 218-221.

2. Eberhart CG, Brat DJ, Cohen KJ, Burger PC. Pediatric neuroblastic brain tumorscontaining abundant neuropil and true rosettes. Pediatr Dev Pathol 2000; 3: 346352.

3. MacDonald TJ. Aggressive infantile embryonal tumors. J Child Neurol 2008; 23: 1195-1204.

4. Judkins AR, Ellison DW. Ependymoblastoma: dear damned, distracting diagnosis, farewell! Brain Pathol 2008; Epub ahead of print: doi: 10.1111/j.1750-3639. 2008.00253.x.

5. Gessi M, Giangaspero F, Lauriola L et al. Embryonal tumors with abundant neuropil and true rosettes. A distinctive CNS primitive neuroectodermal tumor. Am J Surg Pathol 2009; 33: 211-217.

6. Dunham C, Sugo E, Tobiad V, Wills E, Perry A. Embryonal tumor with abundant neuropil and true rosettes (ETANTR). Report of a case with prominent neurocytic differentiation. J Neurooncol 2007; 84: 91-98.

7. Jay V, Edwards V, Halliday W, Rutka J, Lau R. "Polyphenotipic" tumors in the central nervous system: problems in nosology and classification. Pediatr Pathol Lab Med 1997; 17: 369-389.

8. Rodriguez FJ, Scheithauer BW, Giannini C, Bryant SC, Jenkins RB. Epithelial and pseudoepithelial differentiation in glioblastoma and gliosarcoma: a comparative morphologic and molecular genetic study. Cancer 2008; 15: 2779-2789.

9. Seeger RC, Brodeur GM, Sather H et al. Association of multiple copies of the N-myc oncogene with rapid progression of neuroblastomas. N Engl J Med 1985; 31: 1111-1116.

10. Aldosari N, Bigner SH, Burger PC et al. and MYCN oncogene amplification in medulloblastoma. A fluorescence in situ hybridization study on paraffin sections 
from the Children's Oncology Group. Arch Pathol Lab Med 2002; 126: 540-544.

11. Takei H, Nguien Y, Mehta V, Chintagumpala M, Dauser RC, Adesina AM. Low-level copy gain versus amplification of myc oncogenes in medulloblastoma: utility in predicting prognosis and survival. Laboratory investigation. J Neurosurg Pediatr 2009; 3: 61-65.

12. Pfister S, Remke M, Castoldi $\mathrm{M}$ et al. Novel genomic amplification targeting the microRNA cluster at $19 q 13.42$ in a pediatric embryonal tumor with abundant neuropil and true rosettes. Acta Neuropathol 2009; 117: 457-464.

13. Fuler C, Fouladi M, Gajjar A, Dalton J, Sanford RA, Helton KJ. Chromosome 17 abnormalities in pediatric neuroblastic tumor with abundant neuropil and true rosettes. Am J Clin Pathol 2006; 126: 277-283.

14. Matlashewski GJ, Tuck S, Pim D, Lamb P, Schneider J, Crawford LV. Primary structure polymorphism at amino acid residue 72 of human p53. Mol Cell Biol 1987; 7: 961-963.

15. Boltze C, Roessner A, Landt O, Szibor R, Peters B, Schneider-Stock R. Homozygous proline at codon 72 of p53 as a potential risk factor favoring the development of undifferentiated thyroid carcinoma. Int $J$ Oncol 2002; 21: 1151-1154.

16. Papadakis ED, Soulitzis N, Spandidos DA. Association of p53 codon 72 polymorphism with advanced lung cancer: the Arg allele is preferentially retained in tumours arising in Arg/Pro germline heterozygotes. $\mathrm{Br}$ J Cancer 2002; 87: 1013-1018.

17. Furihata M, Takeuchi T, Matsumoto M et al. p53 mutation arising in Arg72 allele in the tumorigenesis and development of carcinoma of the urinary tract. Clin Cancer Res 2002; 8: 1192-1195.

18. Koul D. PTEN signaling pathways in glioblastoma. Cancer Biol Ther 2008; 7: 1321-1325. 